Mon. Not. R. Astron. Soc. 000,116(2010) Printed 3 November $2018 \quad$ (MN IATEX style file v2.2)

\title{
Warp or lag? The ionized and neutral hydrogen gas in the edge-on dwarf galaxy UGC 1281
}

\author{
P. Kamphuis ${ }^{1,2,3 \star}$, R.F. Peletier ${ }^{1}$, P.C. van der Kruit ${ }^{1}$ and G.H. Heald ${ }^{4}$ \\ ${ }^{1}$ Kapteyn Astronomical Institute, University of Groningen, Postbus 800, 9700 AV, Groningen, the Netherlands \\ ${ }^{2}$ Astronomisches Institut der Ruhr-Universität Bochum, Universitätsstr. 150, 44780 Bochum, Germany \\ ${ }^{3}$ Humboldt Research Fellow \\ ${ }^{4}$ Netherlands Institute for Radio Astronomy (ASTRON), 7990 AA Dwingeloo, the Netherlands
}

\begin{abstract}
The properties of gas in the halos of galaxies constrain global models of the interstellar medium. Kinematical information is of particular interest since it is a clue to the origin of the gas. Until now mostly massive galaxies have been investigated for their halo properties.

Here we report on deep $\mathrm{H}$ I and $\mathrm{H} \alpha$ observations of the edge-on dwarf galaxy UGC 1281 in order to determine the existence of extra-planar gas and the kinematics of this galaxy. This is the first time a dwarf galaxy is investigated for its gaseous halo characteristics. We have obtained $\mathrm{H} \alpha$ integral field spectroscopy using PPAK at Calar Alto and deep H I observations with the WSRT of this edge-on dwarf galaxy. These observations are compared to $3 \mathrm{D}$ models in order to determine the distribution of $\mathrm{H} \mathrm{I}$ in the galaxy.

We find that UGC 1281 has $\mathrm{H} \alpha$ emission up to $25^{\prime \prime}(655 \mathrm{pc})$ in projection above the plane and in general a low $\mathrm{H} \alpha$ flux. Compared to other dwarf galaxies UGC 1281 is a normal dwarf galaxy with a slowly rising rotation curve that flattens off at 60 $\mathrm{km} \mathrm{s}^{-1}$ and a central depression in its $\mathrm{H}$ I distribution. Its $\mathrm{H}$ I extends $70^{\prime \prime}(1.8 \mathrm{kpc})$ in projection from the plane. This gas can be explained by either a warp partially in the line-of-sight or a purely edge-on warp with rotational velocities that decline with a vertical gradient of $10.6 \pm 3.7 \mathrm{~km} \mathrm{~s}^{-1} \mathrm{kpc}^{-1}$. The line-of-sight warp model is the preferred model as it is conceptually simpler. In either model the warp starts well within the optical radius.
\end{abstract}

Key words: UGC 1281, Galaxies: halos, Galaxies: ISM, Galaxies: spiral, Galaxies: structure, Galaxies: kinematics and dynamics, Galaxies: dwarf

\section{INTRODUCTION}

The discovery of a very extended H i halo in NGC 891 has shown that $21 \mathrm{~cm}$ observations are a powerful tool to study the structure and kinematics of gaseous halos. Since this discovery (Swaters et al. 1997) many investigations have followed studying this (Oosterloo et al. 2007) and other galaxies. Investigations at $21 \mathrm{~cm}$, as well as other wavelengths, have shown that NGC 891 is not a special case in having a gaseous halo (Schaap et al. 2000; Lee et al. 2001; Rossa \& Dettmar 2003; Barbieri et al. 2005; Westmeier et al. 2005; Boomsma et al. 2008).

It is thought that a large part of this halo gas is brought up from the disk by galactic fountains (Shapiro \& Field 1976; Bregman 1980). In this model supernovae explosions expel the hot hydrogen gas from the disk into the halo. In

* E-mail:peter.kamphuis@astro.rub.de the halo the gas cools down and condenses before returning to the disk. Studies of massive galaxies have shown that this mechanism is very likely at work in several galaxies, either inferred from the fact that the extra-planar $\mathrm{H}$ I is concentrated towards the disk (Oosterloo et al. 2007) or that the majority of high velocity clouds are located near the bright inner disk (Boomsma et al. 2008).

The gas in the halo of NGC 891 shows rotational velocities that are lower then the velocities of the gas in the disk. This 'lag' is observed in H I (Fraternali et al. 2005) as well as in $\mathrm{H} \alpha$ (Heald et al. 2006; Kamphuis et al. 2007) and determined to be linear with a magnitude of $\sim 16 \mathrm{~km} \mathrm{~s}^{-1}$ $\mathrm{kpc}^{-1}$ in the vertical direction.

Simulations have shown that the lag cannot be explained by galactic fountains alone (Fraternali \& Binney 2008). Together with other observational facts, such as the low metallicity of the High Velocity Clouds (HVCs) and the presence of filaments and other irregular gaseous structures in the 
halo (Sancisi et al. 2008), this has given rise to the idea that the halo must also be partially formed from gas that has been accreted from the Intergalactic Medium (IGM) or companion galaxies. However, the gradients by themselves do not necessarily require accretion from the IGM (Beniamin 2002; Barnabè et al. 2006).

Even though this lagging behavior is observed in other galaxies then NGC 891 - e.g. Heald et al. (2007) studied two other large galaxies and found a lag in both of themit is not known whether it occurs in all gaseous halos. Until now, kinematical studies have focussed on massive galaxies and one Low Surface Brightness (LSB) galaxy (Matthews \& Wood 2003) but dwarf galaxies have not yet been investigated for a kinematic lag.

Here we present deep $\mathrm{H}$ I and $\mathrm{H} \alpha$ observations of the edge-on dwarf galaxy UGC 1281 and analyze the kinematics of the $\mathrm{H}$ I and $\mathrm{H} \alpha$ in and above the plane. The $21 \mathrm{~cm}$ line emission was observed with the Westerbork Synthesis Radio Telescope (WSRT) and the $\mathrm{H} \alpha$ with the Integral Field Unit (IFU) PPAK on the $3.5 \mathrm{~m}$ telescope at Calar Alto.

The edge-on orientation of UGC 1281 provides us with an excellent opportunity to study the vertical structure of gas in a dwarf galaxy. Our H I observations of UGC 1281 are some of the deepest observations ever conducted on a dwarf galaxy, and never before has an edge-on dwarf galaxy been observed to this depth.

UGC 1281 is a nearby edge-on dwarf galaxy $\left(\mathrm{M}_{\mathrm{B}}=-\right.$ 15.8 , Swaters \& Balcells (2002)) with a systemic velocity of $156 \mathrm{~km} \mathrm{~s}^{-1}$ at a distance of $5.4 \mathrm{Mpc}$ (Karachentsev et al. 2004). The galaxy has a angular diameter of $4.46^{\prime}\left(\mathrm{D}_{25}\right)$ (de Vaucouleurs et al. 1992) on the sky which would translate to $7 \mathrm{kpc}$ at the given distance. van Zee (2000, 2001) measured the star formation rate in UGC 1281 to be very low $\left(0.0084 \mathrm{M}_{\odot} \mathrm{yr}^{-1}\right)$. However, this should be considered as a lower limit since the $\mathrm{H} \alpha$ flux was not corrected for internal extinction. These observations are supported by the non-detections in radio continuum (Hummel et al. 1991) and IRAS. Indeed, Rossa \& Dettmar (2003) observed UGC 1281 as a part of their extra-planar diffuse ionized gas (DIG) survey and could not detect any extra-planar $\mathrm{H} \alpha$ in this dwarf galaxy. All these observations indicate a very low star formation rate in UGC 1281.

Under the assumption that gaseous halos are created by processes related to star formation, little to no halo is expected when the SFR is low. However, in a dwarf galaxy gas might easily escape from the disk due to the weak potential. Also, even though small galaxies are not expected to accrete baryonic matter at lower redshifts (Hoeft \& Gottloeber 2010), UGC 1281 is in the transition region, between accreting and non-accreting galaxies, and therefore a modest amount of lagging extra-planar gas might be present if the vertical gradient is predominantly formed by the accretion of matter.

This article is structured as followed. In $\S 2$ we will describe the observations and data reduction. $\S 3$ will contain the results of the observations followed by a presentation of our models in $\S 4$ We will discuss our results in $\S 5$ and summarize in $\S 6$.

\begin{tabular}{ll}
\hline Parameter & Value \\
\hline Observation date & 2004 September \\
Total length of observation $(\mathrm{hr})$ & $4 \times 12$ \\
Velocity center of band $\left(\mathrm{km} \mathrm{s}^{-1}\right)$ & 156 \\
Total bandwidth $(\mathrm{MHz})$ & 10 \\
Channels in obs. & 1024 \\
Channel sep. in obs. $(\mathrm{kHz})$ & 9.77 \\
Channels in final cube & 61 \\
Vel. res. after Hanning smoothing $\left(\mathrm{km} \mathrm{s}^{-1}\right)$ & 8.24 \\
\hline
\end{tabular}

Table 1. Log of the H I observations.

\section{OBSERVATIONS \& DATA REDUCTION}

\subsection{Radio Data}

The $21 \mathrm{~cm}$ line emission, or $\mathrm{H} \mathrm{I}$, observations were obtained with the WSRT during four nights in September 2004. In total 4 complete $12 \mathrm{hr}$ observations were performed using the Maxi-Short configuration. This configuration gives the optimum performance for imaging extended sources. This is because it provides a good sampling of the inner $U-V$ plane with a shortest base line of $36 \mathrm{~m}$. The longest baseline is $2754 \mathrm{~m}$. An overview of the observational parameters is given in Table 1 .

The data were reduced using the MIRIAD package (Sault et al. 1995). Care was taken not to include data affected by solar or other interference. Before and after each $12 \mathrm{hr}$ observation a calibration source (3C147 and CTD93) was observed, thus enabling us to determine the spectral response of the telescope. During each $12 \mathrm{hr}$ observation no additional (phase) calibration sources were observed, as is standard practice with the WSRT. Due to the large bandwidth, the time variations can be determined from self-calibration of detected sources in the continuum image constructed from the channels free of line emission. In this case UGC 1281 itself was not detected in continuum emission.

After the reduction and calibration additional analysis was performed with the GIPSY package (van der Hulst et al. 1992). The final cube was reduced to 61 velocity channels with a velocity spacing of $4.12 \mathrm{~km}$ $\mathrm{s}^{-1}$, which results in a velocity resolution after Hanning smoothing of $8.24 \mathrm{~km} \mathrm{~s}^{-1}$. The original cube has a spatial resolution of $25.0^{\prime \prime} \times 13.4^{\prime \prime}$. This cube was smoothed to two cubes with circular beams of $26^{\prime \prime}(0.68 \mathrm{kpc})$ and $60^{\prime \prime}(1.57$ $\mathrm{kpc})$ to avoid beam orientation effects and to detect low level emission (see Table 2).

For the calculation of the minimum detectable column in Table 2 we used a velocity width of $16.5 \mathrm{~km} \mathrm{~s}^{-1}$, because real emission will never appear in a single channel.

The position of the center of the galaxy, as determined by Swaters \& Balcells (2002) from $R$-band photometry, was set to zero in the three cubes and they were rotated by $50^{\circ}$ $\left(\mathrm{PA}=40^{\circ}\right.$, Swaters \& Balcells (2002) $)$ to orient the major axis of the galaxy parallel to the $\mathrm{x}$-axis of the image. 


\begin{tabular}{lccc}
\hline Parameter & Full Resolution & Circular Beam & Low Resolution \\
\hline Spatial resolution (') & $25.0 \times 13.4$ & $26.0 \times 26.0$ & $60.0 \times 60.0$ \\
Beam size (kpc) & $0.65 \times 0.35$ & $0.68 \times 0.68$ & $1.57 \times 1.57$ \\
rms noise per channel (mJy beam $\left.{ }^{-1}\right)$ & 0.44 & 0.50 & 0.72 \\
Minimum detectable column density $\left(3 \sigma ; \mathrm{cm}^{-2}\right)$ & $7.1 \times 10^{19}$ & $4.0 \times 10^{19}$ & $1.1 \times 10^{19}$ \\
\hline
\end{tabular}

Table 2. Parameters of the H I Data Cubes.

\begin{tabular}{lll}
\hline Parameter & Field 1\&2 & Field 3 \\
\hline Name & North\&Center & South \\
Observation date & 2006 September & same \\
Exposure time (s) & 3600 & 1800 \\
Central wavelength $(\AA)$ & 6273 & 6273 \\
Total bandwidth $(\AA)$ & 1628 & 1628 \\
Channels in obs. & 1050 & 1050 \\
Channel sep. in obs. $(\AA)$ & 1.55 & 1.55 \\
Channel separation $\left(\mathrm{km} \mathrm{s}^{-1}\right)$ at $\mathrm{H} \alpha$ & 70.8 & 70.8 \\
\hline
\end{tabular}

Table 3. $\log$ of the $\mathrm{H} \alpha$ observations.

\section{$2.2 \quad$ IFU data}

The $\mathrm{H} \alpha$ was observed with the PPAK integral field unit on the $3.5 \mathrm{~m}$ telescope at Calar Alto (Kelz et al. 2006). Three positions were observed in one night (see Table 3). At each position several exposures were taken. Two positions were observed for $3 \times 1200 \mathrm{~s}$ (North and Center) and one for $3 \times$ $600 \mathrm{~s}$ (South). The lower exposure time of the Southern field was due to twilight. The pointings of the North and South fields were shifted by $\sim \pm 76^{\prime \prime}$, compared to the Center, along an axis with a PA slightly offset $\left(\sim 10^{\circ}\right.$ offset $)$ from the PA of the galaxy. This was done to ensure a good coverage of extra-planar $\mathrm{H} \alpha$. The spectra cover a wavelength range $\sim 5500$ - $7000 \AA$ with a resolution of $4.1 \AA(187 \mathrm{~km}$ $\mathrm{s}^{-1}$ at $\mathrm{H} \alpha$ ). The conditions were partially photometric with a mean seeing of $1.3^{\prime \prime}$, which is much less than the fiber size $\left(2.7^{\prime \prime}\right)$.

For the reduction of the PPAK spectra the IRAF package was used. The steps in the dohydra Guide (Valdes 1995) were followed manually to ensure complete control over the reduction. The only deviation taken from the steps as described in this guide was that we traced the apertures on the science frames themselves. This was done because there was more than enough continuum emission in each spectrum and there was no need to introduce additional errors due to shifting the apertures.

For the initial wavelength calibration 7 lines of a HeNe+ThAr lamp were used. Afterwards a fine tuning calibration was performed with 5 sky lines in each science frame. For the sky line subtraction a total of 95 sky fibers from the three pointings were used. All sky fibers were checked for inconsistencies and, except for sky fibers with the galaxy in their field of view, none were found.

After the reduction and wavelength calibration the different exposures of each field were combined by calculating the median of the separate exposures. No clipping was applied. The three fields were then positioned into one field by overlaying the continuum images of the fields on top of a $R$ band DSS image. By taking special care that the five stars in the three fields were aligned properly, this procedure resulted in a position error less than $1^{\prime \prime}$, which is smaller than the $2.7^{\prime \prime}$ fiber size.

Our reduced and calibrated data were further analyzed with a combination of IDL programs produced by the SAURON collaboration (e.g. de Zeeuw et al. (2002)) and ourselves. The data were Voronoi binned with the voronoi_2d_binning IDL program (Cappellari \& Copin $2003)$ to obtain a higher $\mathrm{S} / \mathrm{N}$ ratio $(\mathrm{S} / \mathrm{N}>20)$. Like the $\mathrm{H}$ I, the central position of the galaxy was set to $(0,0)$ and the galaxy was rotated by $50^{\circ}$ to align the major axis with the $\mathrm{x}$-axis.

\section{RESULTS}

Here, we first discuss the distribution and kinematics of the hydrogen that can be directly derived from the data. In Section [5] we will compare the distributions and kinematics of the ionized and neutral gas, highlight the differences and similarities, and discuss possible interpretations of these distributions.

\subsection{Gas distribution}

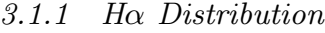

Fig. 1 shows the $\mathrm{H} \alpha$ flux obtained with the PPAK IFU instrument. The flux in each bin was determined by fitting a Gaussian, with the IDL routine GAUSSFIT, to an average of the spectra in each bin. The area under this Gaussian is then considered to be the flux in each bin. All the fitted Gaussians were inspected by eye, and none of the fitted lines showed significant deviations from Gaussian. For display purposes the log values of the flux are displayed. For the same reason the bins without emission are simply taken away from the data and thus not displayed. Overlaid on the image are the contours of an integrated H I (zeroth moment) map.

The first thing we see from Fig. 1] is that the ionized hydrogen is mostly located in 5 or 6 distinct peaks but that there is low level emission almost everywhere in the central field of view. The distinct peaks can most likely be associated with large H I r regions in the galaxy whereas the low level emission is indicating a diffuse ionized component. The two innermost peaks at the NE and SW side of the center of the galaxy might be caused by a ring-like structure or central depression in the distribution. This seems to be implied by their equal distance from the center; however, they could also be normal H I I regions.

A warp is clearly seen in the H I regions as well as the diffuse emission. The peak of the emission starts to deviate 


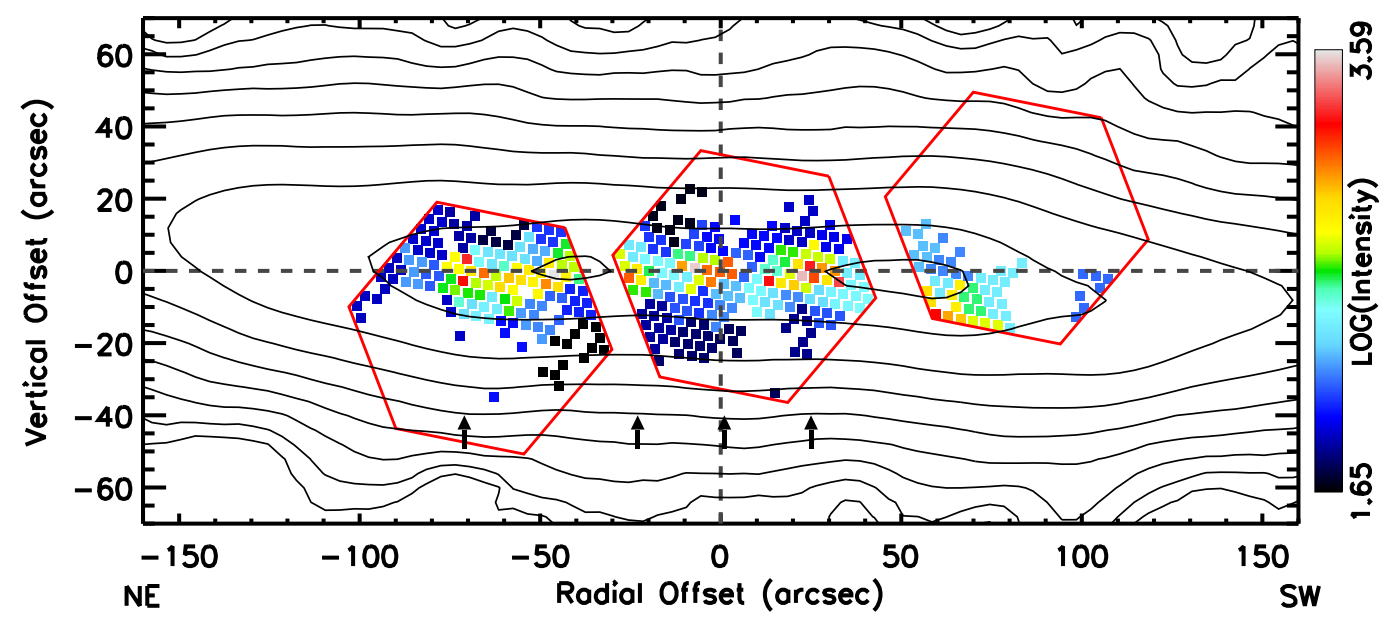

Figure 1. Integrated $\mathrm{H} \alpha$ flux map of UGC 1281. The map was constructed by taking the log of the area under the fitted Gaussian in each spectrum. The red lines indicate the full field of view of the PPAK observations. Overlaid in black are the contours of an integrated H I (zeroth moment) map. The contours are at 1.5, 3, 6, 12, 24, 48, 96, 192, $273 \sigma$ levels of the data where $\sigma=2.2 \mathrm{mJy}^{\mathrm{beam}}{ }^{-1}$. The arrows indicate the positions of the velocity cuts parallel to the minor axis in Fig. 6

from the major axis at a radial distance of $\sim 50^{\prime \prime}(1.3 \mathrm{kpc})$. In the South West (SW) of the galaxy we see an exception to this behavior with a large ionized hydrogen peak below the major axis, where the diffuse emission still seems to be mostly on the major axis. This seems to indicate an H I r region that is either somewhat offset from the plane or located in the outskirts of the galaxy. If the warp is partially along the line of sight the outer parts will not only experience a change in position angle but also in inclination. Thus if this H I r region is located in the outskirts of the galaxy its position can be in the (warped) plane of the galaxy.

The $\mathrm{H} \alpha$ distribution could, in theory, be severely affected by internal dust extinction, especially in the edge-on orientation. However, in the case of UGC 1281 this seems unlikely because no clear dust lane can be observed in HST imaging of UGC 1281 Bomans \& Weis 2008) and dwarf galaxies are expected to have a low metallicity (Pilvugin et al. 2004) and therefore little dust content. The reddish color of UGC 1281 (Makarova \& Karachentsev 1998) is most likely caused by the absence of a large young stellar population. This once more confirms the idea that UGC 1281 has a low SFR.

In our data the maximum distance to the mid-plane where diffuse gas is still detected is $\sim 25^{\prime \prime}(0.65 \mathrm{kpc})$. This is similar to the the extent of the stars $\left(\frac{1}{2} d_{25, \text { minor }}=18^{\prime \prime}\right.$, van Zee (2000), $\frac{1}{2} d_{25, \text { minor }}=23^{\prime \prime}$, de Vaucouleurs et al. (1992) ). If we fit an exponential to the inner vertical intensity profile of the ionized gas we find a scale height of $8.5^{\prime \prime}(0.22 \pm 0.03 \mathrm{kpc})$ assuming that the galaxy is seen perfectly edge-on. When we follow the same procedure for the continuum emission in our spectra and an $I$-band image 1 we find a scale height of $7.6^{\prime \prime}(0.20 \pm 0.01 \mathrm{kpc})$ and $8.0^{\prime \prime}(0.21 \pm 0.01 \mathrm{kpc})$ respectively.

\subsubsection{H I Distribution}

Fig. 2] shows the DSS 2 red image of UGC 1281 overlaid with the contours of our integrated H I flux map. This H I map was constructed by adding all the channels of the Circular Beam data cube. To keep the addition of noise to a minimum, only regions that are above $3 \sigma$ in the Low Resolution cube (see Table 21) were considered.

From Fig. 2 we can see that the H I of UGC 1281 is at first glance quite symmetrically and evenly distributed. However, a closer look reveals asymmetries and peculiarities in the $\mathrm{H}$ I distribution. It warps away from the major axis at about $90^{\prime \prime}(2.4 \mathrm{kpc})$ on the South West and at about $100^{\prime \prime}(2.6 \mathrm{kpc})$ on the North East. The warp initially shows the normal S-shape observed in many edge-on galaxies (García-Ruiz et al. 2002) but bends back to the plane of the inner disk at larger radii. This behaviour is seen especially at the South West side, at a radial offset of $\sim 200^{\prime \prime}$. This warp was already observed by García-Ruiz et al. (2002) in the WHISP observations of this galaxy (van der Hulst et al. 2001).

When we compare the lowest contour in the integrated moment map of the WHISP observations with our own (see Fig. 2. red contour), we see that in our observations more emission is detected in the radial as well as in the vertical direction. The growth in both directions is similar in extent and this indicates that even with our deep observations we might not be detecting the lowest levels of emission of this galaxy.

Furthermore, the H I distribution displays a central depression. This depression appears as a ring around the center of the galaxy and ranges from $10^{\prime \prime}$ to $40^{\prime \prime}(0.26-1.05 \mathrm{kpc})$ radial offset from the center of the galaxy. It appears to be symmetrical around the center of the galaxy.

The H I in UGC 1281 shows significant extensions away from the major axis. The $\mathrm{H}$ I extends up to $70^{\prime \prime}(1.8 \mathrm{kpc})$ on both sides of the plane at column densities $N_{H I}=$ $4.0 \times 10^{19} \mathrm{~cm}^{-2}(3 \sigma)$. This extent is much more than the FWHM of the beam $\left(26^{\prime \prime}\right)$ which is clearly seen in Fig. 3 


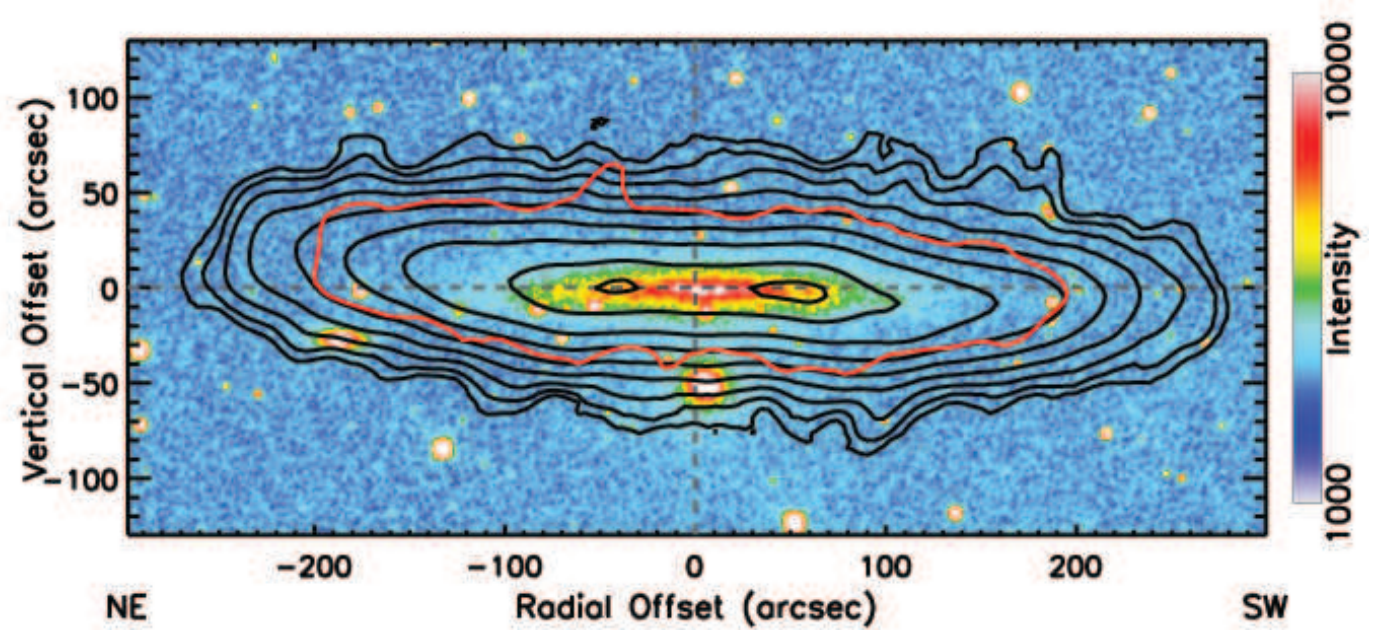

Figure 2. Continuum image in the red from the DSS 2 overlaid with contours of our integrated H I flux map of UGC 1281 (see text). The black contours are the same as in Fig. 1] The red contour indicates the $3 \sigma$ contour of the WHISP observations.

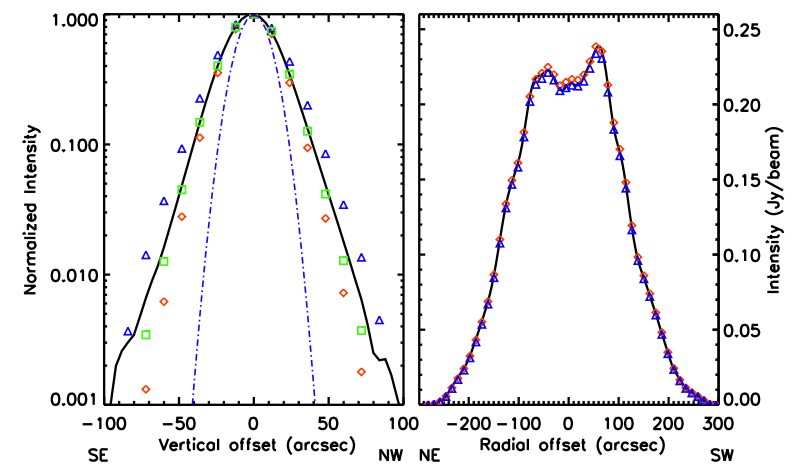

Figure 3. Intensity profiles of the observed H I. Left figure: Vertical intensity profile, averaged over the inner $100^{\prime \prime}$ in the radial direction and normalized to the peak intensity. Right figure: Radial intensity profile, averaged over the inner $40^{\prime \prime}$ of the galaxy in the vertical direction. Black line: Data, Green squares: Best fit model, Red diamonds (Blue triangles): best fit model with a scale height $-2^{\prime \prime}\left(+4^{\prime \prime}\right)$ (see $\S$ 4). Blue dotted dashed line: beam.

This figure shows the vertical distribution of the data (black solid line) and a Gaussian (blue dot-dashed line) with a FWHM of $26^{\prime \prime}$. Both are normalized to the maximum of the data. In this figure it is easily observed that the wings of the data are much more extended than the observational beam.

\subsection{Velocity Distribution}

\subsubsection{Ho Velocities}

Fig. 4 shows the velocity field of the PPAK observations. This velocity field was obtained by taking the peak position of the Gaussian fitted to each bin (see $\S 3.1 .1$. This is by no means equal to the actual deprojected maximum rotational velocity in the galaxy but it is an apparent mean velocity determined by a combination of the rotational velocity, the

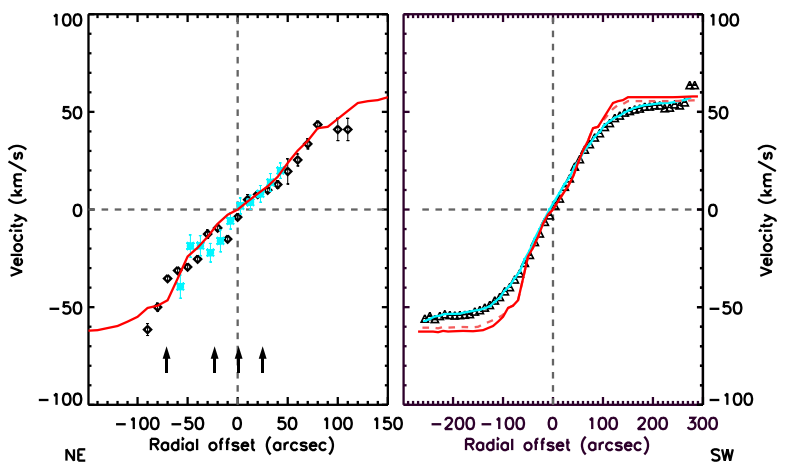

Figure 5. Velocities along the major axis. The left panel shows the $\mathrm{H} \alpha$ velocities: Black symbols are the PPAK data presented in this paper, blue symbols are the data obtained by Kuzio de Narav et al. (2006). Right figure shows H I velocities: Black symbols are the $\mathrm{HI}$, the blue line shows the velocities obtained from the model. The red lines are the input unprojected rotation curves of the best fit models described in $\S 4$ The arrows indicate the positions of the velocity cuts parallel to the minor axis in Fig. [6

density distribution of the ionized gas, and the opacity of the dust. From here on whenever we mention velocity we are referring to this mean velocity unless otherwise noted. The gaussian fitting procedure, and therefore the mean velocity, was chosen because with a channel separation of 70 $\mathrm{km} \mathrm{s}^{-1}$ it is impossible to confidently fit the intrinsic shape of the emission line.

Fig. 5 (left) shows a cut $10^{\prime \prime}$ wide of the velocity field along the major axis. Overplotted are the velocities obtained by Kuzio de Naray et al. (2006) with the DensePAK IFU (blue symbols) and the rotation curve obtained from the modeling (see § 4). Kuzio de Narav et al. (2006) were not able to trace emission as far out in radius with their observations. Since their exposure time and fiber size is equal to ours this is most likely caused by the fact that they do not bin the data and the lower sensitivity of the DensePak IFU. The ve- 


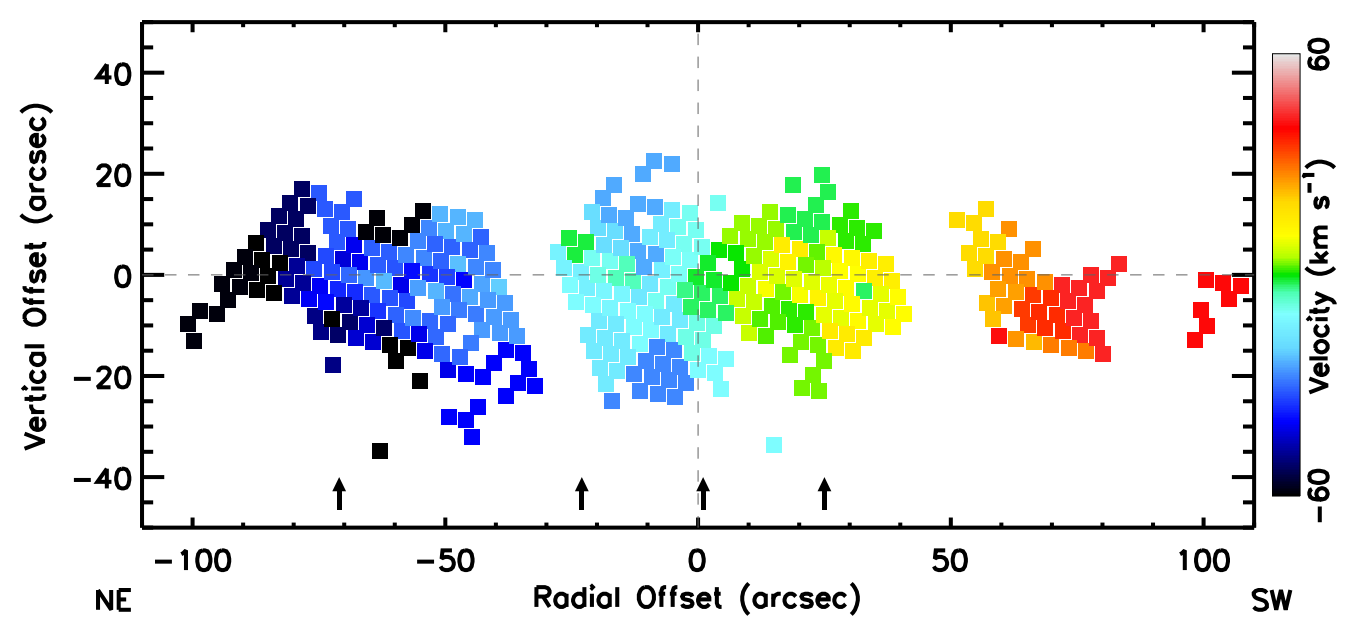

Figure 4. Velocity field of the ionized gas. The field was constructed by taking the central position of the fitted Gaussian in all binned spectra. The systemic velocity $\left(\mathrm{V}_{\mathrm{sys}}=156 . \mathrm{km} \mathrm{s}^{-1}\right)$ has been set to 0 . The separate pixels show the fiber positions and the colors run from -60 to $60 \mathrm{~km} \mathrm{~s}^{-1}$. The arrows indicate the positions of the velocity cuts parallel to the minor axis in Fig. 6]

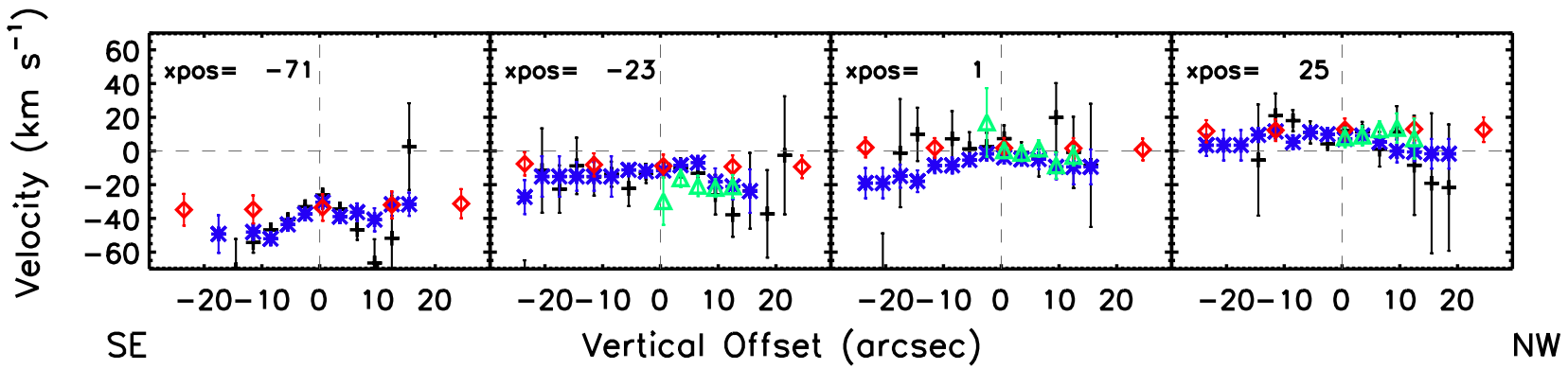

Figure 6. Four cuts parallel to the minor axis through the velocity field at $-71,-23,1,25^{\prime \prime}$ radial offset from the center. The negative (positive) offsets are the South East (North West) in the sky. Black points are the unbinned PPAK data. Blue, voronoi binned PPAK data. Red, H i data. Green, Kuzio de Narav et al. (2006) data.

locities obtained by Kuzio de Naray et al. (2006) agree well with our values, which assures us that there are no systematic errors in our reduction or the gaussian fitting procedure.

In this plot we see clearly that the part of the galaxy observed in our $\mathrm{H} \alpha$ field of view is still resembling a slow rising rotation curve that indicates solid body rotation. This behavior of the rotation curve is quite typical for dwarf galaxies, which all seem to have a large inner region in which their rotation curve resembles solid body rotation (Côté et al. 2000). This behavior could be caused by us considering the mean velocities. However, this is unlikely as we will discuss in $\S 3.2 .2$,

Another thing that is quite clear is that the flux peaks in the $\mathrm{H} \alpha$ along the major axis stand out in the velocity curve as areas with lower velocities (Fig. 5, left, black arrows). This is expected if they are H I r regions, since the chance that they would lie on the line of nodes is low, and would imply that most of the peaks we see in the $\mathrm{H} \alpha$ distribution are indeed H I r regions. Another possible explanation is that the intensity peaks correspond to a higher density in the radial density profile of the galaxy with thicker clumps offset from the line perpendicular to the line of sight. This explanation is supported by the fact that some of the peaks appear symmetrically around the center of the galaxy (see Section 3.1.1) and that the star formation rate in UGC 1281 is so low $\left(\mathrm{SFR}=0.0084 \mathrm{M}_{\odot} \mathrm{yr}^{-1}\right)$ that giant $\mathrm{H}$ I I regions would not be expected to reside in the galaxy.

If so, this would require that as the influence of the overdensity or H I I region, on the shape of the line profile diminishes, the measured velocities should increase. We can see that this is the case when we look away from the mid-plane as is illustrated in Fig. 6] These plots show the velocity as function of vertical offset from the plane in bins $12^{\prime \prime}$ wide for the binned and unbinned $\mathrm{H} \alpha$ (blue and black, respectively), the data from Kuzio de Narav et al. (2006) (green), and the $\mathrm{H}$ I (red) at the positions of the peaks 2 Here we see that the velocities move away from the systemic velocity as we look higher above the plane. This confirms the idea that the velocities on the major axis are lowered by a line-of-sight projection effect. These results and their implications will be discussed in Section 5.2 in combination with the results of the $\mathrm{HI}$.

\footnotetext{
2 Note that also above the major axis, the DensePak data and our data agree within the errors.
} 


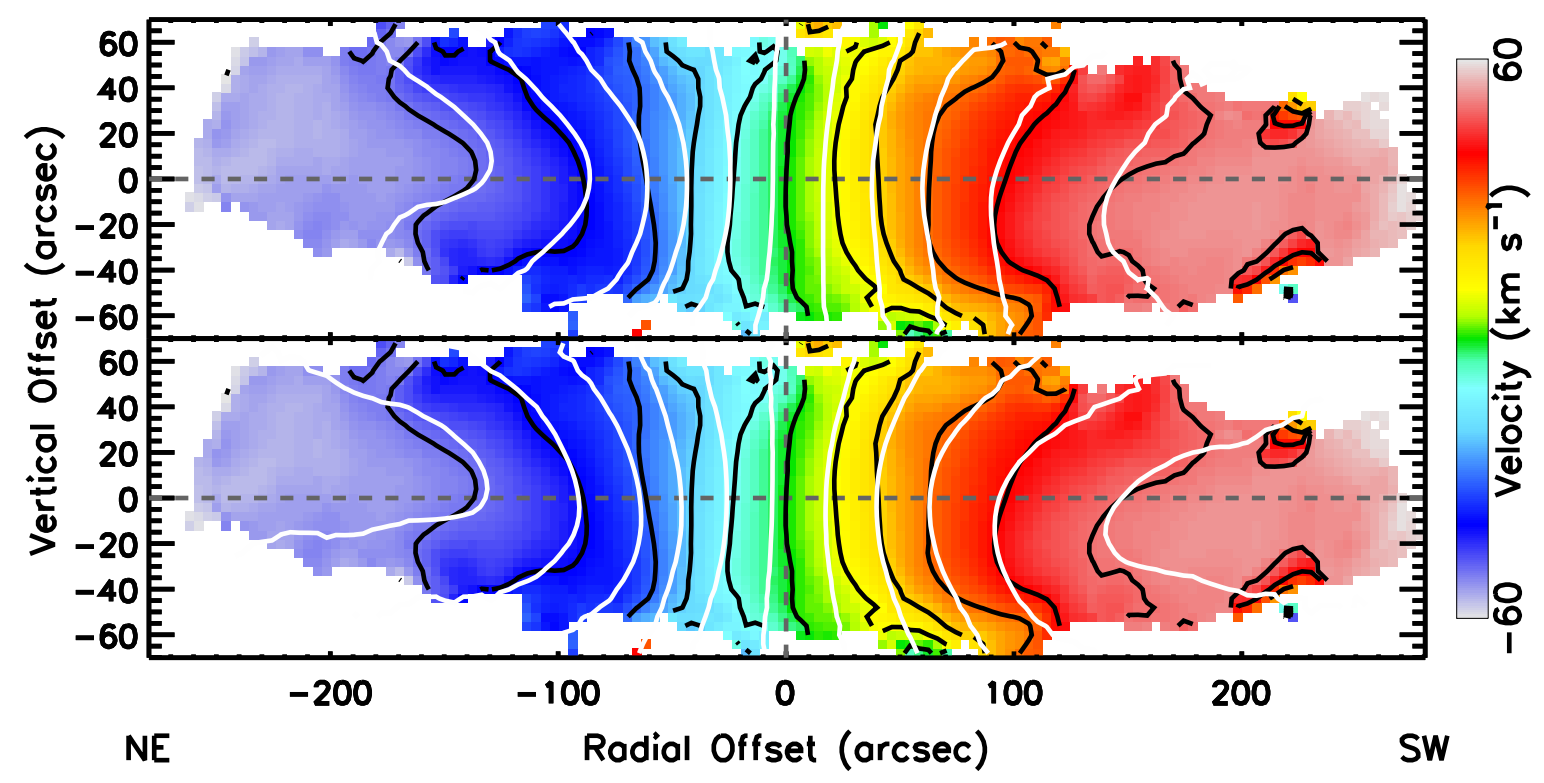

Figure 7. Velocity field of the neutral hydrogen. The field was constructed with the task MOMENTS in GIPSY (see text). Contours are from $-60 \mathrm{~km} \mathrm{~s}^{-1}$ to $60 \mathrm{~km} \mathrm{~s}^{-1}$ with increasing steps of $10 \mathrm{~km} \mathrm{~s}^{-1}$. Black contours are the data. The white contours in the upper panel show the line-of-sight warp model. In the lower panel the white contours show the best fit edge-on model with a lag of $10.6 \mathrm{~km}$ $\mathrm{s}^{-1} \mathrm{kpc}^{-1}($ see $\S$ ).

\subsubsection{H I Velocities}

Fig. 7 shows the velocity field of the $\mathrm{H}$ I observations. This velocity field was constructed with the MOMENTS routine in GIPSY by selecting the first moment of the data cube. The routine determines the intensity weighted mean velocity position of the peak of the line profile in every pixel of the cube. For symmetric profiles, this is analogous to fitting a gaussian profile to the emission line and taking its velocity at the peak. Since UGC 1281 has only small rotational values the line profiles are almost symmetric. We have checked this by comparing a map with the velocities where the line profiles have their maximum with this GIPSY map and we find no differences greater than $6 \mathrm{~km}$ $\mathrm{s}^{-1}$, which is less than the velocity resolution (see Table 1). Thus, these velocities are in principle comparable one to one with the velocities of the $\mathrm{H} \alpha$ and differences should be due to the conditions of the gas (distribution effects, dust, resolution, self absorption, or a real difference in the rotational speed of the ionized and neutral gas).

It is common to retrieve the rotation curve of edge-on galaxies through envelope tracing. Theoretically this is the correct way of retrieving the rotation curve of an edge-on galaxy (Sancisi \& Allen 1979), however there are several reasons why Gaussian fitting is preferable in an edge-on dwarf system. As explained in the previous paragraph the velocities in a dwarf galaxy are small, this means that the dispersion of the gas makes up a significant part of the line profile. Since in envelope tracing, either by fitting gaussians or a scaling of the maximum intensity (Sofue \& Rubin 2001), this dispersion is a chosen parameter it increases the uncertainty of envelope tracing. Furthermore, envelope tracing is also an estimation of the real rotational velocities and detailed modeling is still required. Therefore we prefer the well understood and described method of Gaussian fitting. This does mean that only from the modeling we obtain information about the real rotation curve.

Fig. 5 (right) shows a cut $10^{\prime \prime}$ wide of the velocity field along the major axis. Here we see the same slow rise in the rotation curve as seen in the $\mathrm{H} \alpha$ but we also see that in the $\mathrm{HI}$ we do reach the flat part of the rotation curve at $\sim 120^{\prime \prime}$ radial offset, outside the $\mathrm{H} \alpha$ field of view, from the center of the galaxy at a maximum velocity $\sim 60 \mathrm{~km} \mathrm{~s}^{-1}$.

If we move away from the plane (Fig. 7 and 8) we see that the velocities are lower than on the major axis. This implies that either the outer parts of the galaxy are heavily inclined, that the disk is flaring or that the gas above the plane is 'lagging'. We can quantify this vertical velocity gradient in normalized PV diagrams (see Kamphuis et al. (2007), § 6.2) parallel to the minor axis (Fig. 8, bottom row) by fitting a straight line to the maxima between $20^{\prime \prime}$ and $50^{\prime \prime}$ offset from the plane. Of course this fit is affected by the warp, lag, beam smearing and possibly other systematics. Therefore, the measured value has to be compared to the same measurements of models that can explain the observed declining velocities. These models will be presented in the next section and discussed in Section 5

\section{MODELS}

The $\mathrm{H} \alpha$ observations show such an irregular distribution that constructing a model for comparison will not bring more insight into the structure of the $\mathrm{H} \alpha$ distribution. Also, the distribution is peaked in many places whereas our models would resemble a smooth exponential distribution. We therefore decided to forego any attempt at modeling the ionized hydrogen in 3D. However, there are several parameters that can be derived from the data or obtained from the 


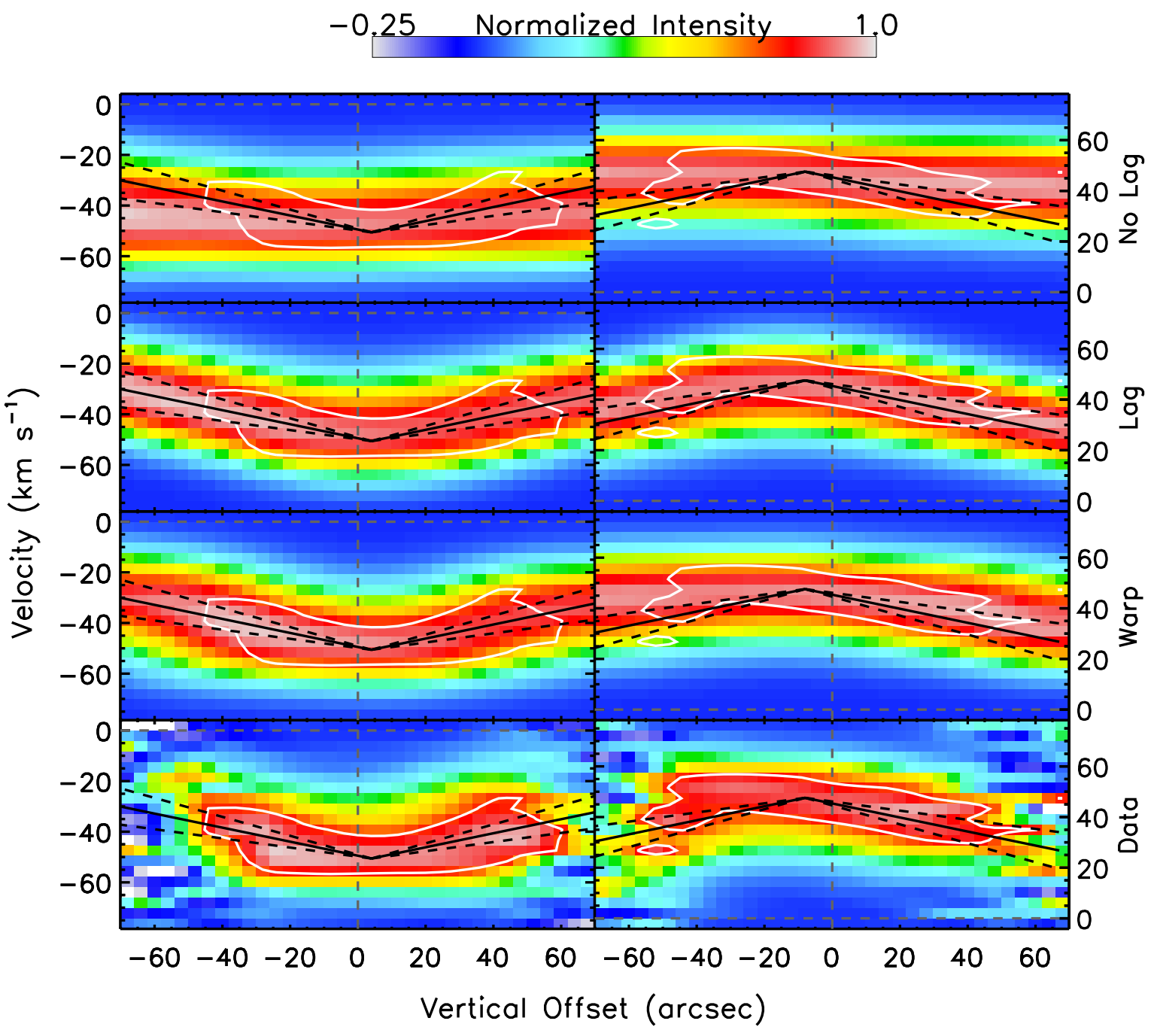

Figure 8. Normalized PV diagrams parallel to the minor axis averaged over $100^{\prime \prime}$ at a radial offset of $-175^{\prime \prime}$ to $-75^{\prime \prime}($ left $)$ and $75^{\prime \prime}$ to $175^{\prime \prime}$. From top to bottom, the edge-on model without lag, the edge-on model with lag, the line-of-sight warp model and the data. The white contour outlines the 0.75 level of the data. The solid lines show the lag obtained from an edge-on lagging model (see $\S 4.1$ ). The dashed lines show the derived error on the lag. The zero point is set to the point of maximum intensity in a non-normalized PV diagram.

\begin{tabular}{lll}
\hline Parameter & Value & Reference \\
\hline EM Scale height & $0.22 \pm 0.03 \mathrm{kpc}$ & {$[1]$} \\
Scale length & $1.09 \mathrm{kpc}$ & {$[2]$} \\
SFR & $0.0084 \mathrm{M} \odot \mathrm{yr}^{-1}$ & {$[2]$} \\
$\mathrm{H} \alpha$ luminosity & $3.81 \pm 0.01 \times 10^{-13} \mathrm{erg} \mathrm{s}^{-1} \mathrm{~cm}^{-2}$ & {$[3]$} \\
\hline
\end{tabular}

Table 4. H $\alpha$ parameters that could be measured from the observations or obtained from the literature. [1] this paper, [2] van Zee (2001), [3] van Zee (2000).

literature. These are presented in Table 4 for comparison to the H I model.

\subsection{Tilted ring models}

To better understand the 3D distribution and the kinematics of the neutral hydrogen in UGC 1281 we have constructed a range of tilted ring models. These models were created with the GIPSY task GALMOD. The GALMOD routine was modified by one of us (G. H.) and F. Fraternali to be able to include radial and vertical motions as well as a vertical gradient for the rotational velocities.

The modeling is not straightforward, since many different parameters may be independently fitted and some of them are degenerate. In the case of an edge-on galaxy matters are even more complicated because small changes in density in the outer rings can seriously affect the inner rings of the model. For this reason the fitting always has to start at the outer radii and then move inward ring by ring. Since it is clear that the warp is not symmetric at all, we fit both sides of the galaxy independently. The goodness of a fit was determined by investigating several representations of the data (PV diagrams, residual maps) by eye.

The following, iterative, procedure was followed for the fitting. We start by comparing radial and vertical density profiles of the data with the models (see Fig. 3). This has to 


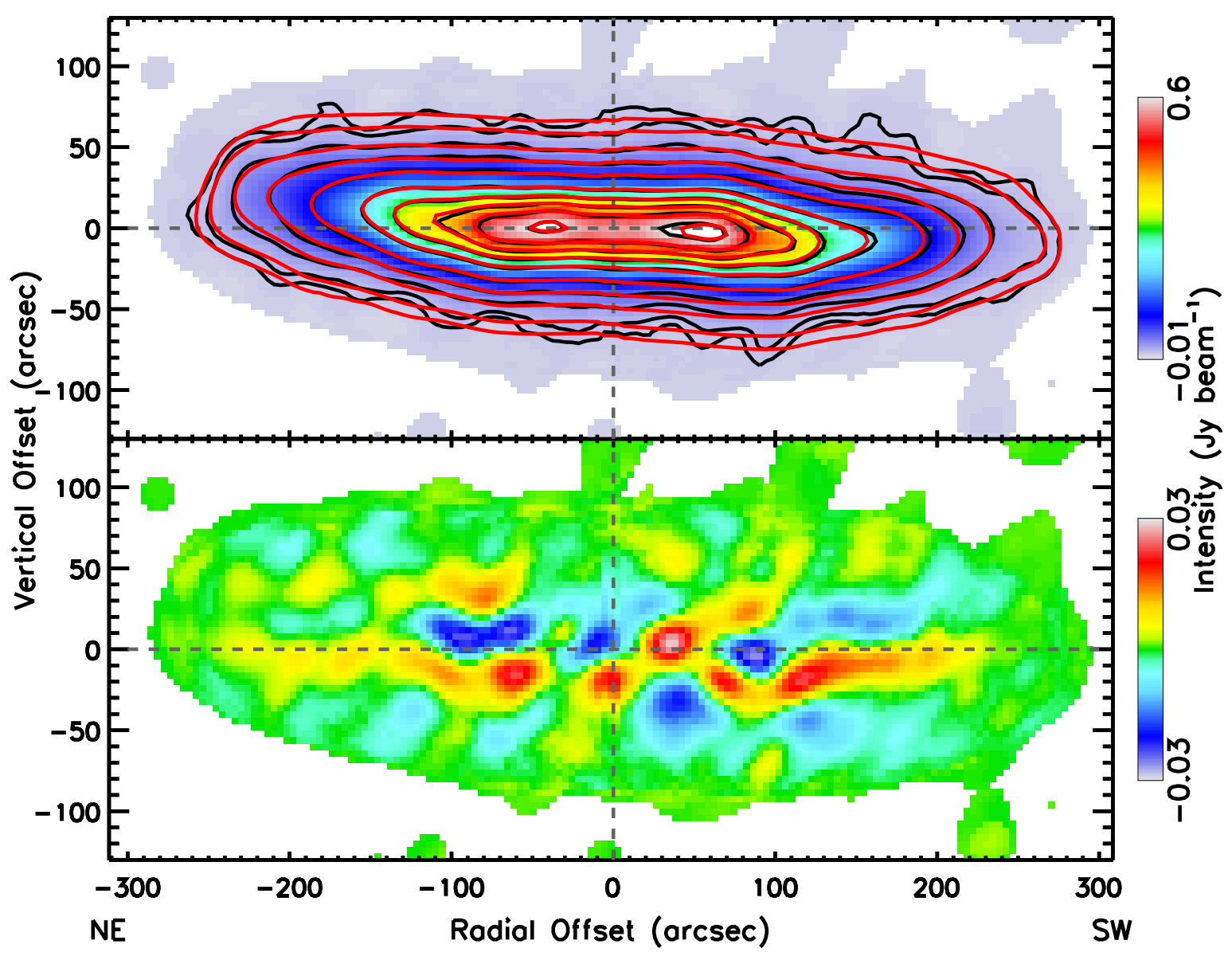

Figure 9. Upper panel: Integrated H i flux map of UGC 1281 (see Fig. 2). The black contours are the data at the same levels as in Fig. 1 The red contours are the same levels for the best fit edge-on model. Lower panel: Residual map of the data minus the best fit edge-on model (total intensity).

be done to get a initial guess of the density profile and scale height. When these profiles have a reasonable fit we start fitting the position angle on a integrated moment map. The position angle was deemed to fit when, at all radii, the vertical position of the peak value was equal in the models and the data.

In the tilted ring model the inclination of each ring is related to the position angle and dependent on the angle between the axis on which the ring tilts and the line of sight. We assume a simple warp, where the tilt axii for all rings are aligned, e.g. a straight line of nodes. The assumption of a straight line of nodes is a simplifying one that lowers the degrees of freedom in the model, making it possible to constrain the vertical distribution and kinematics in the model. By assuming a simple warp the inclination of each ring is coupled to its position angle by just one parameter, the angle between the line of sight and the line of nodes. We shall refer to this angle as the warp axis angle.

From the data it is impossible to determine an exact warp axis angle. We therefore set out to find the maximum and minimum warp axis angle that can fit the observations. Starting at a minimum warp axis angle of $0^{\circ}$, e.g. all rings $90^{\circ}$ inclined, we found an acceptable density distribution immediately.
We then set out to find the maximum warp axis angle. This maximum is set at the point where the vertical profile starts to show a break, which is not observed in the data (see Fig. 3). By slowly increasing the warp axis angle and comparing the vertical distribution of the model to the data we find that this break becomes significant when the warp axis angle is greater than $60^{\circ}$, thus defining the maximum line-of-sight warp model to have a warp axis angle less than $60^{\circ}$.

At this stage we split our modeling into two parts. Besides a model with a warp axis angle of $0^{\circ}$, the edge-on model, we construct a second model with a warp axis angle of $55^{\circ}$, the maximum line-of-sight warp model. Now the only parameter of the density that is not determined yet is the vertical distribution of each ring. In our models the vertical density, of all the rings, declines as an exponential of which the steepness is set by its scale height. By increasing/decreasing the scale height of our models systematically we find the upper and lower limit of the scale height. We allow the scale height to change from ring to ring but only increase toward larger radii. This increase would resemble a flare. In this way we find that the best fit scale height of the $\mathrm{H}$ I in UGC 1281 is a flaring model that ranges from $10^{\prime \prime}$ to $15^{\prime \prime}(0.26$ to $0.39 \mathrm{kpc})$ for our edge-on model and from $7^{\prime \prime}$ to 
-0.002 Intensity (Jy Beam ${ }^{-1}$ ) $\quad 0.092$

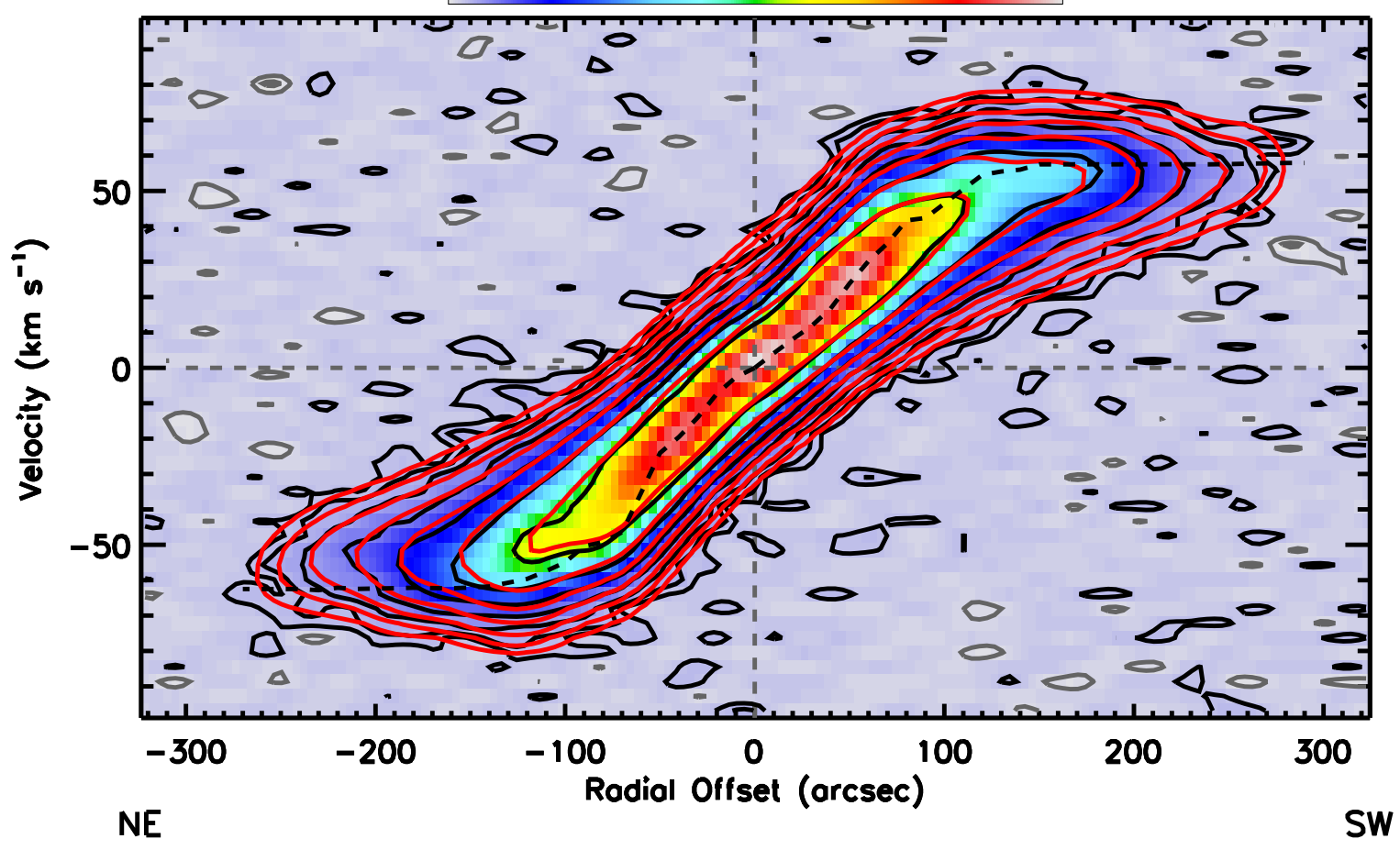

Figure 10. Color plot of the H I PV diagram along the major axis. Overlaid with contours of the data (grey/black) and the best fit model (red). The contour levels are $-3 \sigma,-1.5 \sigma, 1.5 \sigma, 3 \sigma$ and $6 \sigma(1 \sigma=0.5 \mathrm{mJy} /$ beam $)$ etc.
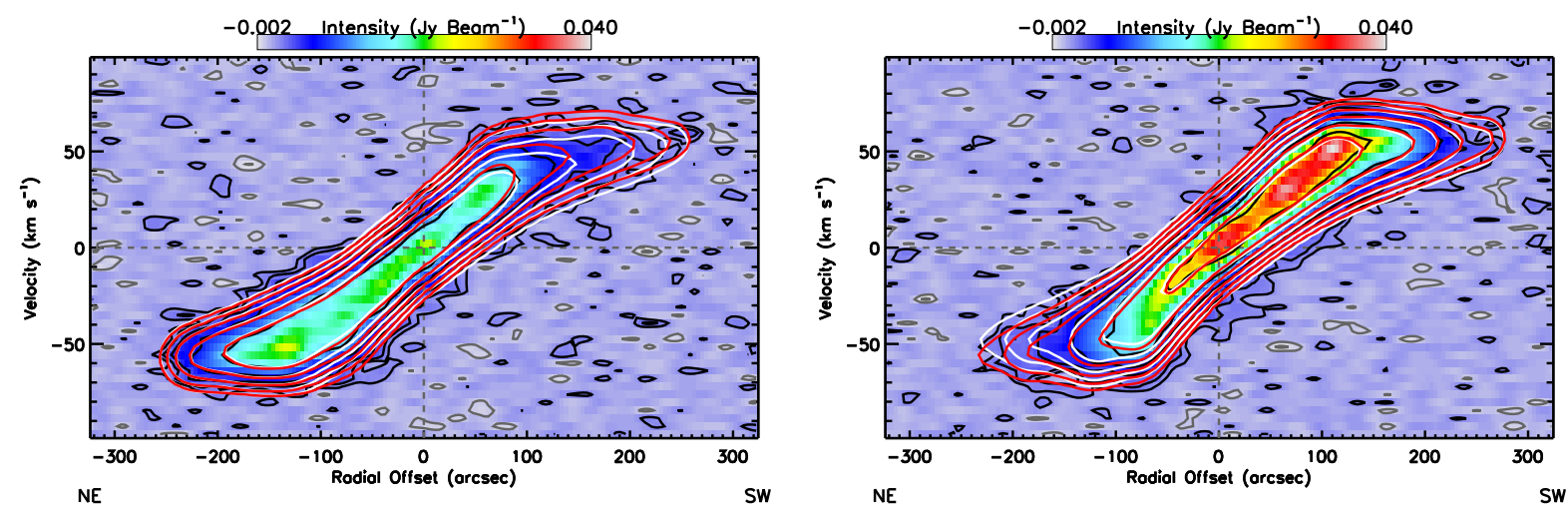

Figure 12. Left (Right): Color plot of the H I PV diagram at $26^{\prime \prime}\left(-26^{\prime \prime}\right)( \pm 0.6 \mathrm{kpc})$ offset from the major axis. Contours are at $-3 \sigma,-$ $1.5 \sigma, 1.5 \sigma, 3 \sigma, 6 \sigma$ and so on. The black solid contour is the data, red contour is the best fit model with no lag, white contour is the best fit model with an assumed vertical gradient of $10.6 \mathrm{~km} \mathrm{~s}^{-1} \mathrm{kpc}^{-1}$ in the rotation curve. The colormap ranges are indicated above the panel in Jy/beam.

$12^{\prime \prime}(0.18$ to $0.31 \mathrm{kpc})$ for our maximum line-of-sight warp model.

In order to estimate the accuracy of our scale heights we systematically raise and lower the best fit flaring model. It turns out that the vertical profile significantly deviates from the observations when we add (subtract) more then $4^{\prime \prime}\left(2^{\prime \prime}\right)$ (100 and $50 \mathrm{pc}$ respectively) to the scale heights of our best fit models. The vertical distributions of these models are shown in Fig. 3 as blue triangles (upper limit) and red diamonds (lower limit) for the edge-on model. The blue dot-dashed line indicates the spatial resolution $(\mathrm{FWHM}=$ $\left.26^{\prime \prime}\right)$.
As a last check on the density distribution we construct an integrated moment map of the residual cube. Fig. 9 shows the residual map of the best fit density distribution. This figure shows only the density distribution of the best fit edge-on model because, above the sensitivity limit of the data, the differences in the density distribution between the two models are less than $5 \%$. Therefore the difference between these two models would not be visible in such plots. In this figure the wiggles that necessitate adding a warp, purely in PA, to the edge-on model, and their reproduction in this model, are clearly seen.

After we obtain a satisfactory density distribution we 

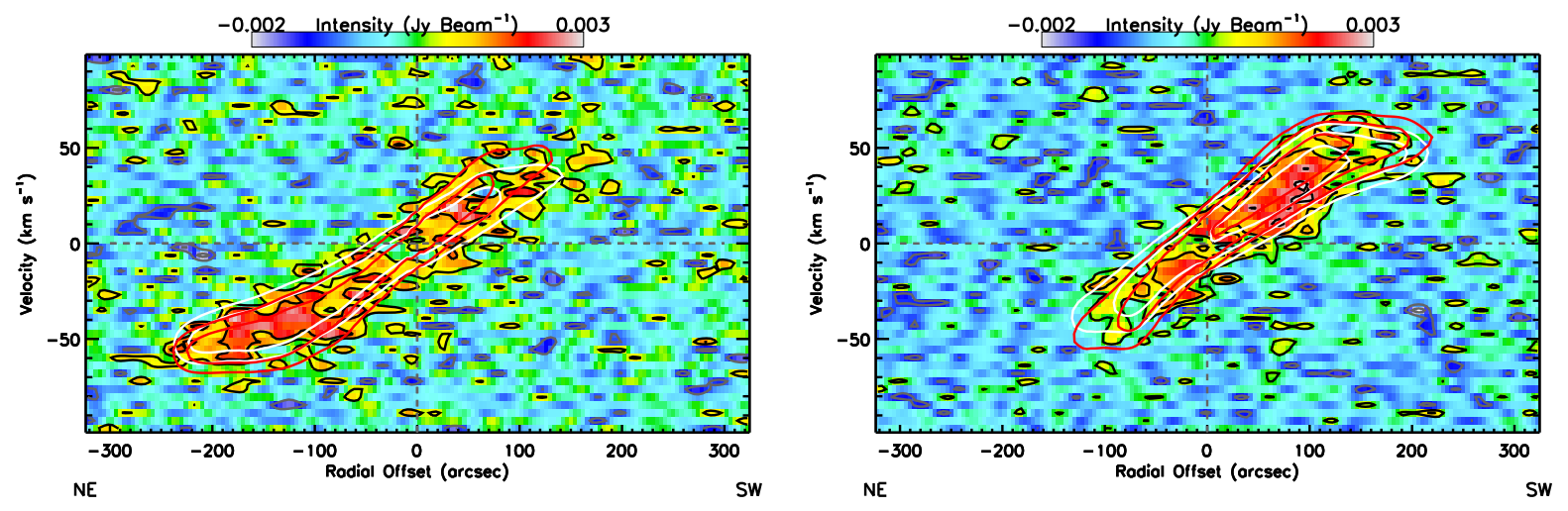

Figure 13. Same as Fig. 12 but now at $\pm 56^{\prime \prime}( \pm 1.3 \mathrm{kpc})$ offset from the plane.

start fitting the rotation curve and dispersion of the gas. Initially we set the dispersion of the gas to be constant at 9 $\mathrm{km} \mathrm{s}^{-1}$ and then start fitting the rotation curve. As a first guess for a lower limit of the rotation curve we use the velocities from the $\mathrm{H} \alpha$ and $\mathrm{H}$ I. For the inner $\pm 50^{\prime \prime}$ we use the $\mathrm{H} \alpha$ velocities and beyond this point we use the velocities obtained from the H I first moment map (see Fig. 5). This rotation curve is then raised from the outside in until it fits the data.

As an upper limit we start from a flat rotation curve and fit this curve by lowering the velocities from the inside out. The change between rings is always half of the difference between the previous two rings, with a minimal increase of $4 \mathrm{~km} \mathrm{~s}^{-1}$ for the inner rings. Because a low inner density could artificially lower the observed velocities in an edge-on system we have set the densities of the inner rings to zero while fitting this rotation curve.

Once more the fitting is an iterative process where the goodness of the fit is determined by comparing the major axis position-velocity (PV) diagram of the data to the model. An example of such an PV diagram is shown in Fig. 10, where the best fit edge-on model is plotted with the data. The color scheme and the black contours are the data whereas the red contours display our best fit edge-on model. Again only the edge-on model is shown because also in this PV diagram the differences between the two models are less than $5 \%$. When we have obtained satisfactory fits to this PV diagram for both the upper and lower limit rotation curves we find that they differ no more then a channel width $\left(4.1 \mathrm{~km} \mathrm{~s}^{-1}\right)$ at any ring position which indicates that the rotation curve is well constrained. Besides the upper and lower limit rotation curves matching very well, also the rotation curves of both, independently fitted, sides of the galaxy match up quite well. The final rotation curves are shown in Fig. 5] and 14 (Top Panel). These curves are the average of the upper and lower limit of both best fit models. Fig. 10 show the rotation curve of the best fit edge-on model as a black dashed line overlaid on the major axis PV diagram.

After fitting the rotation curve we found that the fit to the major axis PV diagram could be significantly improved by introducing a gradient in the velocity dispersion. This gradient runs from $\sigma_{\mathrm{v}}=11 \mathrm{~km} \mathrm{~s}^{-1}$ in the center to $\sigma_{\mathrm{v}}=8 \mathrm{~km}$ $\mathrm{s}^{-1}$ at the largest radii (see Fig. 14, Bottom Panel).

The model cubes should now be comparable to the data cube everywhere and any major deviations can only be caused by gas that is deviating from co-rotation at high projected distances from the mid-plane. In the case of the maximum line-of-sight warp model the observations are fully reproduced by the best fit model. However, in the case of the edge-on model the velocities above the plane are clearly over estimated. This can be seen in Figure 8 When we compare the edge-on model (Upper panels) to the data (Lower panels) it is easily seen that in the data the emission peaks at a vertical offset lie at lower velocities than the emission peak in the mid-plane. However, such a bending is only produced by the warp when the rings are not edge-on and thus completely lacking in the edge-on model without a lag. Therefore we reproduce the edge-on model with a rotation curve that declines as a function of distance to the plane, e.g. a lag. We construct seven models where the vertical gradient is increased from 4 to $28 \mathrm{~km} \mathrm{~s}^{-1} \mathrm{kpc}^{-1}$ in steps of $4 \mathrm{~km} \mathrm{~s}^{-1}$ $\mathrm{kpc}^{-1}$.

When we measure the vertical gradient in these models in the same way as in the data (see $\S 3.2 .2$ ), we find that the value measured from the data corresponds to a lag of $10.6 \pm 3.7 \mathrm{~km} \mathrm{~s}^{-1} \mathrm{kpc}^{-1}$ (see Fig. 11) in the edge-on model. After adding this lag to the model it was found that, due to beam smearing effects, the major axis PV-diagram showed emission at slightly lower velocities than the data and the line-of-sight warp model. To correct for this effect the flat part of the input rotation curve was raised by $2 \mathrm{~km} \mathrm{~s}^{-1}$. With this correction the major axis PV-diagrams of both models agreed within $5 \%$ again. A model with this lag produces a satisfactory fit at all heights.

As a last check to the model we compare the non-lagging model to the data and the lagging edge-on model in PV diagrams at two distances above (below) the mid-plane. Figures 12 and 13 show PV diagrams parallel to the major axis at \pm $26^{\prime \prime}$ and $\pm 56^{\prime \prime}$ offset from the plane. Already Fig. 12 is suggesting that a model with a lag gives a better fit, though we cannot exclude the non-lagging model at this height. At a height $\sim 60^{\prime \prime}$ above the plane (Fig. 13) it is clearly seen that the velocities around the $3 \sigma$ contours are too high in the non-lagging model.

Figure 8 shows also the normalized PV diagrams parallel to the minor axis for the best fit lagging edge-on model. Also in these figures it is now seen that the edge-on model with a vertical gradient and the maximum line-of-sight warp model fit the data equally well.

Fig. 14 shows the parameters for the best fit models 


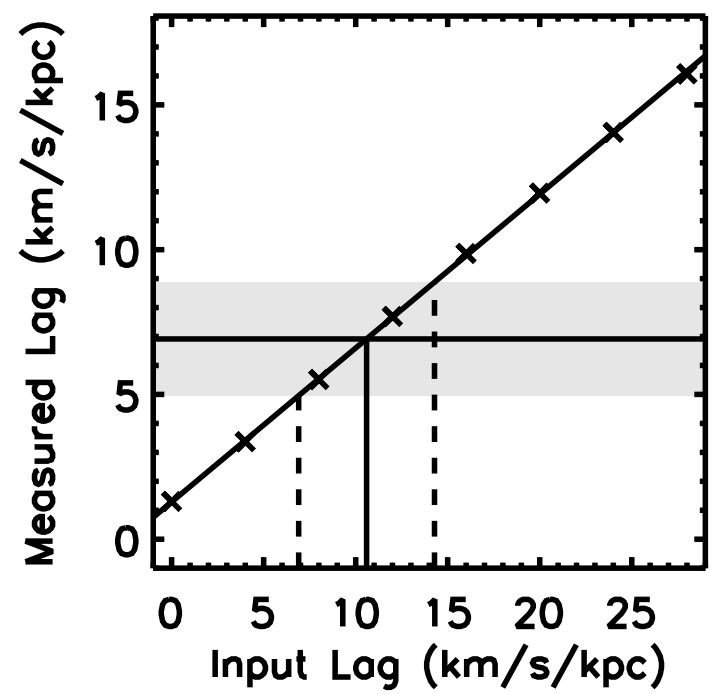

Figure 11. Input lag vs. Measured lag in the models. The solid line shows the fit to the measured values (displayed by crosses) of the models. The horizontal solid line displays the value obtained from the data. The vertical solid line shows where the measured value intersects the model fit. The grey shaded area indicates the error in the measurement and the dashed vertical lines where the error intersects the fit. This range determines the error bar on the value of the lag.

of UGC 1281 (Edge-on model (black lines) \& Line-of-sight warp model (symbols)). The best fit for the edge-on model has a flare and the scale height ranges from $10^{\prime \prime}(0.26 \mathrm{kpc})$ in the inner parts to a maximum of $15^{\prime \prime}(0.39 \mathrm{kpc})$ on the North Eastern outer parts. The change in PA is the same for both models but in the case of the line-of-sight warp model this is coupled to a change in inclination of the ring as previously explained. The scale length of this model galaxy is $46^{\prime \prime}(1.2$ $\mathrm{kpc})$. The scale length is the same for the maximum warp model, which is also flaring and has a scale height of $7^{\prime \prime}(0.18$ $\mathrm{kpc})$ in the inner parts and $12^{\prime \prime}(0.31 \mathrm{kpc})$ in its outer parts.

\subsection{Ballistic Models}

To investigate what we would expect for UGC 1281 in the sense of extra-planar gas brought up from the disk by supernovae we have used the ballistic model of Collins et al. (2002). In this model gas is blown out of the disk into the halo, with an initial vertical velocity (called the kick velocity). The model naturally predicts a vertical gradient in rotational velocity which has a higher magnitude in model with a high kick velocity. To obtain the vertical gradient of the model we follow the procedure outlined by Heald et al. (2007), with the following difference. The initial disk for the model is infinitely thin, this is because the scale height of the $\mathrm{H} \alpha$ in UGC 1281 is already comparable to the scale height normally used for the initial disk $(0.2 \mathrm{kpc})$. Therefore this model should be thought of as an absolute upper limit on the kick velocity.

We find that the scale height of $0.2 \mathrm{kpc}$ is reproduced

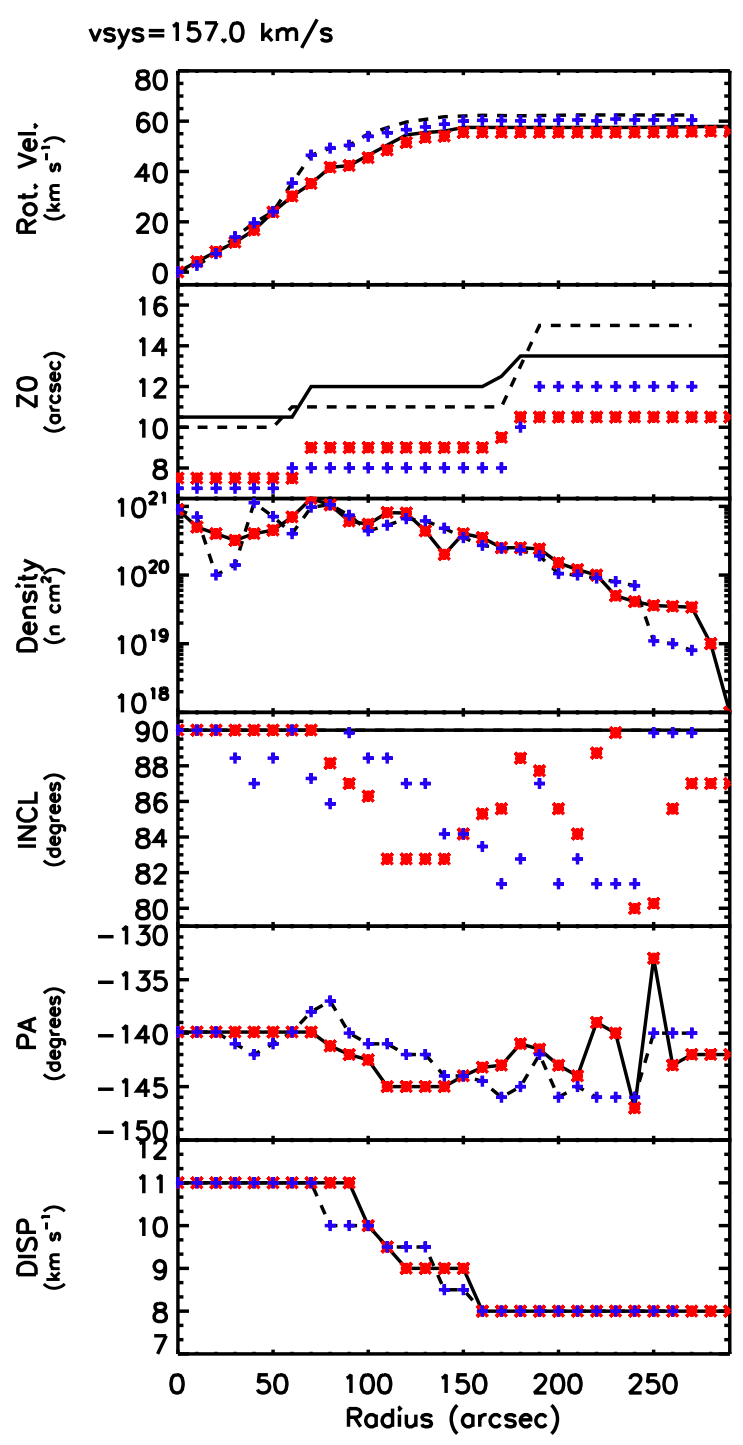

Figure 14. Model parameters as function of radius. For the edgeon model (Solid line: South West side, Dashed line: North East side) and the maximum line-of-sight warp model (Blue plusses: South West side, Red crosses: North East side).

when the initial kick velocity is $10 \mathrm{~km} \mathrm{~s}^{-1}$. We run the simulation until the system reaches steady state ( $\sim$ Gyr). At this point there are no clouds in the halo at radii smaller than $4 \mathrm{kpc}$ due to the radial outward movement of the clouds. Therefore we cannot measure the vertical gradient in the rotational speed at these radii. However, at radii between $5-10 \mathrm{kpc}$ we find a small gradient of $0.4 \mathrm{~km} \mathrm{~s}^{-1} \mathrm{kpc}^{-1}$. This is a very low value as one would expect for a small galaxy like UGC 1281 since kick velocities must be small in a low potential, because otherwise the scale height becomes too large. 


\section{DISCUSSION}

\subsection{Hydrogen in the plane}

When we compare the parameters of the best fit $\mathrm{H}$ I models to the parameters extracted from the $\mathrm{H} \alpha$ data (Table 4) we see that compared to the ionized gas the neutral gas is much more extended in the radial direction. Apart from this its scale length is slightly larger. So, the ionized gas is more centrally concentrated than the $\mathrm{HI}$.

Note that both the neutral gas and the ionized gas display a central depression, or peaks symmetrically around the center, in their distribution (see Section 3.1). Also, the $\mathrm{H} \alpha$ distribution is more irregular than the neutral hydrogen. This can easily be seen in Figures 1 and 2 by counting peaks in the distribution (see Section 3.1.1). One of these 'peaks' in $\mathrm{H} \alpha$ has a clear offset from the plane of the galaxy and this region does not stand out in the $\mathrm{HI}$ observations.

Another effect is the start of the warp. If we look at the integrated moment maps the neutral gas starts to deviate from the major axis at a radius almost two times larger than the radius where the $\mathrm{H} \alpha$ bends away from the major axis (50" and $90^{\prime \prime}$, respectively). Most likely this is a resolution effect, because when we look at the best fit model for the $\mathrm{H}$ I we see both the $\mathrm{H}$ I and the $\mathrm{H} \alpha$ start to warp around $50^{\prime \prime}(1.3 \mathrm{kpc})$.

When we compare the measured velocities of the neutral and ionized gas they look very similar on the major axis (see Fig. 5). Ideally this should only occur if the sizes of both disks are similar and there is no absorption, self-shielding or clumpiness affecting the emission. We know from the analysis of the $\mathrm{H} \alpha$ that the velocities in the plane are lower than those above the plane in several places. Also, the $\mathrm{H}$ I velocities are slightly lower, due to beam smearing, when compared to the best fit model. Since for the $\mathrm{H} \alpha$ emission we can only look at the rising part of the rotation curve, where the rotational velocities are low, deviations from the real rotational velocities are small. Therefore, it is not unreasonable that both effects provide us with the same velocities since all absolute deviations from the rotational velocities are small. Fig. [5 (Red line) shows us that the real rotation curve, obtained from the modeling, is slowly rising and reaches a flat part around $60 \mathrm{~km} \mathrm{~s}^{-1}$. This means that UGC 1281 has the slow rising rotation curve which is common in dwarf galaxies, but it also shows clear differential rotation.

\subsection{Hydrogen above the plane}

We see (Fig. 1) that the $\mathrm{H} \alpha$ extends less far in the vertical direction than the H I. Despite the fact that a difference in sensitivity may cause us to miss $\mathrm{H} \alpha$ emission at distances further from the plane than $20^{\prime \prime}$, the scale height, measured by fitting a single exponential to the vertical distribution, of the $\mathrm{H} \alpha$ is, equal at best, but most likely smaller than the $\mathrm{H}$ I scale height.

Even though the vertical extent of the $\mathrm{H} \alpha$ is limited and not supplying much information above the plane the $\mathrm{H}$ I extends well above the plane and much can be learned from it. If we assume a constant gas dispersion of $9 \mathrm{~km} \mathrm{~s}^{-1}$ we would expect a scale height of $\sim 400$ pc for the disk, from a simple estimate (Puche et al. 1992). This implies that the gas at large distances from the mid-plane is not an additional component but merely the high-latitude gas of a pressure supported disk. This idea is strengthened by the fact that a ballistic model requires a very low kick-velocity $\left(\sim 10 \mathrm{~km} \mathrm{~s}^{-1}\right)$, of the order of the velocity dispersion in the model, and indicates that the gas at large distances from the mid-plane is not pushed to high latitude by star formation processes. Furthermore, continuum maps constructed from the line-free channels of the $21 \mathrm{~cm}$ observations do not show any continuum emission at the position of UGC 1281 once more confirming the low SFR. This implies that UGC 1281 does not contain a typical halo such as seen in NGC 891. However, also in the case of UGC 1281 the kinematics show that as the distance from the mid-plane increases the projected mean velocities decline (see Fig. 8).

From our modeling we obtain two possible explanations for the high latitude $\mathrm{H}$ I and its declining projected mean velocities. We find that a maximum line-of-sight warp model, where we push the warp as much into the line of sight as the data allows, fits the data equally well as a purely edge-on model with a warp in the plane of the sky and a vertical gradient in its rotation curve of $-10.6 \pm 3.7$ $\mathrm{km} \mathrm{s}^{-1} \mathrm{kpc}^{-1}$. Both models assume a single exponential in the vertical direction and the edge-on model does assume that the radial gas distribution above the plane is similar to that in the disk. In theory the lower velocities could also be caused by an alternate distribution above the disk. However, since the vertical gradient is seen almost everywhere in the observations, it seems unlikely that this is the case unless the gas is in a warp or flare and these possibilities are included in the modeling.

To outline the importance of separating between a lag and a line-of-sight warp we will discuss these two options and their implications in two separate sections below. These discussions are by no means meant as a complete overview of the theory and observations behind extra-planar gas but only to highlight the parts where UGC 1281 can significantly contribute to a better understanding of the theories. For a full and complete review of cold extra-planar gas we refer the reader to Sancisi et al. (2008) and references therein.

\subsubsection{A warp}

The first possibility that can explain the observations is that the gas at large projected distances from the mid-plane in UGC 1281 is located in a simple warp. Warps are quite common in disk galaxies and often they are asymmetric (García-Ruiz et al. 2002) as is observed in UGC 1281. If the $\mathrm{HI}$ and its kinematics in UGC 1281 are to be fully explained by a warp, the warping axis has to have an angle of $55^{\circ}$ with respect to the line of sight.

We would like to point out the very small differences between the edge-on model and the maximum line-of-sight warp model. The maximum difference in inclination between the two models is $10^{\circ}$ but on average $5^{\circ}$ in inclination. This results in a difference in scale height of $\sim 3^{\prime \prime}$ (see Fig. 14). These differences take away the need for a vertical gradient completely. This once more shows that differences between 
line-of-sight warps and lagging halos are very subtle, and that great care must be taken to exclude one of the two options.

When we measure once more the maxima in normalized PV diagrams parallel to the minor axis we find an apparent vertical gradient of $4.9 \pm 1.1 \mathrm{~km} \mathrm{~s}^{-1} \mathrm{kpc}^{-1}$, for the maximum line-of-sight warp model, which is consistent with the measurement from the data $\left(6.9 \pm 2.0 \mathrm{~km} \mathrm{~s}^{-1} \mathrm{kpc}^{-1}\right)$. Therefore there is no need to introduce a lag or other extra kinematical effects into this model.

The best fit model for this case has central scale heights which are similar to those measured from the stars and the $\mathrm{H} \alpha$. This would mean that the stars and the ionized gas hardly extend into the warped outer regions of the disk and that all the $\mathrm{H}$ I at large projected distances from the mid-plane is in the warp and flare. We have already seen that the PA starts changing well within the maximum radius of the $\mathrm{H} \alpha$ as well as inside the optical radius. This is seen for every model, whether it is edge-on or maximum line-of-sight.

The start of the warp could be affected by a slight error in the assumed PA. We have tested this by rotating the integrated H I moment map and the red DSS image (See Fig. 2) by an additional 1 and 2 degrees $\left(\mathrm{PA}=39^{\circ}, 38^{\circ}\right.$ respectively) and plotting the vertical offsets of the peak of the vertical profiles. This shows that for the red optical image the $\mathrm{PA}$ of $40^{\circ}$ gives the flattest central distribution but that the start of the warp in the $\mathrm{HI}$ can be pushed outward by $\sim 15^{\prime \prime}$ by assuming a PA of $39^{\circ}$. This means that the observed start of the warp remains well within the optical radius $\left(\frac{1}{2} \mathrm{D}_{25}=134^{\prime \prime}\right)$ even if the assumed PA is slightly off.

The fact that the warp starts within the optical radius is inconsistent with the findings of van der Kruit (2007) that warps start just beyond the truncation radius of the stellar disk. Even more so, we know from the observed $\mathrm{H} \alpha$ emission that also the ionized gas is slightly warped (see $\S$ 3.1.1). This would imply that if the warp in UGC 1281 is formed by the accretion of gas from the IGM its initial disk was not rigid enough to stabilize the infalling gas (van der Kruit 2007). If so, one would expect a clear difference between the onset of the warp, with respect to its truncation radius, between dwarf galaxies and massive galaxies.

\subsubsection{A lag}

In the case of a warp which is purely in the plane of the sky the modeling indicates a larger scale height of the $\mathrm{HI}$ than the scale heights measured from the stars and the ionized hydrogen. This vertical density distribution can be modeled with a single exponential, however the kinematics indicate the need for a vertical gradient in the rotation curve when the warp is purely perpendicular to the line of sight. As in the case of the super-thin galaxy UGC 7321 (Matthews \& Wood 2003) the origin of this lagging neutral hydrogen gas in a Low Surface Brightness (LSB) dwarf galaxy, with low star formation rates, would be puzzling.

If the high latitude gas in UGC 1281 is lagging this would have some implications to current theory. Heald et al. (2007) have compared the lag, or the vertical gradient in the rotation curve in three massive galaxies. They find tentative evidence that when they scale the lag with the observed $\mathrm{H} \alpha$ scale height 3 this new parameter $\left(d V / d h_{z}\right)$ is roughly constant at about $20 \mathrm{~km} \mathrm{~s}^{-1}$ scale height ${ }^{-1}$. However, the galaxies compared are of similar mass. The results presented in this paper give us now the opportunity to take a tentative look at a class of galaxies with much lower mass. The dynamical mass of UGC 1281 is $6.3 \times 10^{9} \mathrm{M}_{\odot}$, measured at our last point of the rotation curve, as opposed to $\sim 1 \times 10^{11} \mathrm{M}_{\odot}$ for the galaxies in the study by Heald et al. (2007)

Within the picture of a lagging disk, we would have $d V / d h_{z}=4.7 \pm 1.7 \mathrm{~km} \mathrm{~s}^{-1}$ per scale height. This value would be inconsistent with a constant $d V / d h_{z} \sim 20 \mathrm{~km}$ $\mathrm{s}^{-1}$ which was found for the 3 massive galaxies. As shown by the ballistic models ( $\S 4.2)$, the lag expected purely on gravitational grounds would be much shallower than the one than the one that we include in this model. Such a lag would thus require additional effect to be at play such as described by e.g. Beniamin (2002); Barnabè et al. (2006); Fraternali \& Binnev (2008). In any case, star formation in the disk would be of negligible influence on the vertical gradient.

The previous discussion clearly shows the need to unambiguously determine a lag in a small galaxy such as UGC1281 as well as the need for larger sample of galaxies with quantified vertical velocity gradients. In the present case, due to the fact that the addition of a lag to the model of UGC 1281 does not significantly improve the match with the data, we prefer the conceptually simpler line-of-sight warp model.

\section{SUMMARY}

We presented $21 \mathrm{~cm}$ and $\mathrm{H} \alpha$ emission in the edge-on dwarf galaxy UGC 1281. This is the first time such sensitive H I data have been presented for a dwarf edge-on.

The integrated $\mathrm{H} \alpha$ velocity map (Fig. 1) shows a nonsmooth distribution on the major axis with several peaks. One of these peaks is actually located beneath the major axis. It is unclear whether this H I r region is located above the plane of the galaxy or in its warped outer parts.

The integrated H I velocity map (Fig. 2) shows a quite regular distribution with a central depression. This central depression appears to be symmetrical in position around the center but from modeling it follows that it is somewhat deeper on the NE side of the galaxy. Such a central depression is not uncommon for dwarf galaxies.

Furthermore this map shows that UGC 1281 is warped in its outer parts and this warp resembles a "normal" Sshape at its start. However, at large radii the warp bends back towards the inner plane of the galaxy.

For the interpretation of the kinematics of the high latitude $\mathrm{HI}$ gas we constructed velocity maps from the $\mathrm{H} \alpha$ and $\mathrm{H}$ I data. Also 3-D models with a modified version of GALMOD are constructed in GIPSY. This modified version

3 Note that Heald et al. (2007) use electron scale heights instead of the emission measure scale heights. 
enables us to construct kinematic models with a vertical gradient.

The velocities obtained from the data show a slow rise in the inner part. This is also seen in the rotation curve obtained from the modeling and therefore unlikely to be an effect of the $\mathrm{H}$ I distribution or resolution. At about $120^{\prime \prime}$ the rotation curve flattens off to a maximum rotational velocity $\sim 60 \mathrm{~km} \mathrm{~s}^{-1}$. This slow rise is common for dwarf galaxies.

From our modeling we find that our data are not sensitive enough to distinguish between a lag or a line-of-sight warp. Both models fit the data equally well and there is only a small difference between the input parameters of the models. However, the models do start to deviate at emission levels slightly lower than our current sensitivity limit. Therefore one would most likely be able to separate between the two models with deeper observations.

In the case of lagging high latitude gas the low vertical extent and the low flux level of the $\mathrm{H} \alpha$ emission would indicate that this high latitude $\mathrm{H}$ I does not originate from galactic fountains. The $\mathrm{H}$ I scale height implies a normal pressure supported disk and thus no need beyond turbulence for a mechanism to bring it up from the mid-plane. If in such a disk the pressure, of the gas, is solely dependent on the density (e.g. barytropic) one would expect it to be co-rotating. However, we find in our analysis that the high latitude gas in this case has a lag of $10.6 \pm 3.7 \mathrm{~km} \mathrm{~s}^{-1}$ $\mathrm{kpc}^{-1}$ when compared to tilted ring models. This lag could be caused by infalling gas or pressure gradients above the mid-plane.

In the case of a line-of-sight warp the ionized hydrogen and the distribution of the stars would, for the most part, not extend into the warped region of the disk. However, the scale height in the central parts would be the same for the stars, $\mathrm{H} \alpha$ and $\mathrm{H}$.

Regardless of which model fits the data best, maximum line-of-sight warp or edge-on, the warp starts well with the optical radius $\left(\mathrm{D}_{25}=4.46^{\prime}\right.$, de Vaucouleurs et al. (1992)), at a radius of $\sim 50^{\prime \prime}$, which is unlike more massive galaxies (van der Kruit 2007).

The small differences in input parameters between a model with a lag and one with a line-of sight warp show that great care must be taken to distinguish between lagging halos and line-of-sight warps since small changes in the modeling can have a great effect on the velocity field.

Summarising our main conclusions:

- The rotation curve of UGC 1281 is slowly rising in its inner parts and flattens off to a maximum rotational velocity $\sim 60 \mathrm{~km} \mathrm{~s}^{-1}$ at $120^{\prime \prime}(3.14 \mathrm{kpc})$.

- The neutral hydrogen in UGC 1281 is more extended than the stars and the ionized emission in its radial distribution.

- The gaseous warp start well within the optical radius.

- Our observations can be fitted by both a vertical gradient in the rotation curve and a line-of-sight warp. The observations are not sensitive enough to separate between these two options. However, the line-of-sight warp model is conceptually simpler and therefore preferable.

\section{ACKNOWLEDGEMENTS}

We thank R. Kuzio de Naray for making her velocity data available to us. We are grateful to S. Sanchez and A. Guijarro for doing the PPAK observations and M. Verheijen for introducing us to PPAK. Many thanks to T. Oosterloo for help with the H I data reduction and to P. Serra, R.-J. Dettmar, D.J Bomans and our anonymous referees for useful comments, discussion and insights.

\section{REFERENCES}

Barbieri C. V., Fraternali F., Oosterloo T., Bertin G., Boomsma R., Sancisi R., 2005, A\&A, 439, 947

Barnabè M., Ciotti L., Fraternali F., Sancisi R., 2006, A\&A, 446, 61

Benjamin R. A., 2002, in A. R. Taylor, T. L. Landecker, \& A. G. Willis ed., Seeing Through the Dust: The Detection of HI and the Exploration of the ISM in Galaxies Vol. 276 of Astronomical Society of the Pacific Conference Series, The Interstellar Disk-Halo Connection: The Rotation of Extra-planar Gas. pp 201-+

Bomans D. J., Weis K., 2008, in A. de Koter, L. J. Smith, \& L. B. F. M. Waters ed., Mass Loss from Stars and the Evolution of Stellar Clusters Vol. 388 of Astronomical Society of the Pacific Conference Series, Feedback and Clusters in the Sdm Galaxy UGC 1281. pp 395-+

Boomsma R., Oosterloo T. A., Fraternali F., van der Hulst J. M., Sancisi R., 2008, A\&A, 490, 555

Bregman J. N., 1980, ApJ, 236, 577

Cappellari M., Copin Y., 2003, MNRAS, 342, 345

Collins J. A., Benjamin R. A., Rand R. J., 2002, ApJ, 578, 98

Côté S., Carignan C., Freeman K. C., 2000, AJ, 120, 3027

de Vaucouleurs G., de Vaucouleurs A., Corwin Jr. H. G., Buta R. J., Paturel G., Fouque P., 1992, VizieR Online Data Catalog, 7137, 0

de Zeeuw P. T., Bureau M., Emsellem E., Bacon R., Carollo C. M., Copin Y., Davies R. L., Kuntschner H., Miller B. W., Monnet G., Peletier R. F., Verolme E. K., 2002, MNRAS, 329, 513

Fraternali F., Binney J. J., 2008, MNRAS, 386, 935

Fraternali F., Oosterloo T. A., Sancisi R., Swaters R., 2005, in ASP Conf. Ser. 331: Extra-Planar Gas The Extraplanar Neutral Gas in the Edge-on Spiral Galaxy NGC 891. pp 239-+

García-Ruiz I., Sancisi R., Kuijken K., 2002, A\&A, 394, 769

Heald G. H., Rand R. J., Benjamin R. A., Bershady M. A., 2006, ApJ, 647, 1018

Heald G. H., Rand R. J., Benjamin R. A., Bershady M. A., 2007, ApJ, 663, 933

Hoeft M., Gottloeber S., 2010, ArXiv e-prints

Hummel E., Beck R., Dettmar R.-J., 1991, A\&AS, 87, 309

Kamphuis P., Peletier R. F., Dettmar R.-J., van der Hulst J. M., van der Kruit P. C., Allen R. J., 2007, A\&A, 468, 951

Karachentsev I. D., Karachentseva V. E., Huchtmeier W. K., Makarov D. I., 2004, AJ, 127, 2031

Kelz A., Verheijen M. A. W., Roth M. M., Bauer S. M., Becker T., Paschke J., Popow E., Sánchez S. F., Laux U., 2006, PASP, 118, 129 
Kuzio de Naray R., McGaugh S. S., de Blok W. J. G., Bosma A., 2006, ApJS, 165, 461

Lee S.-W., Irwin J. A., Dettmar R.-J., Cunningham C. T., Golla G., Wang Q. D., 2001, A\&A, 377, 759

Makarova L. N., Karachentsev I. D., 1998, A\&AS, 133, 181

Matthews L. D., Wood K., 2003, ApJ, 593, 721

Oosterloo T., Fraternali F., Sancisi R., 2007, AJ, 134, 1019

Pilyugin L. S., Contini T., Vílchez J. M., 2004, A\&A, 423, 427

Puche D., Westpfahl D., Brinks E., Roy J., 1992, AJ, 103, 1841

Rossa J., Dettmar R.-J., 2003, A\&A, 406, 505

Sancisi R., Allen R. J., 1979, A\&A, 74, 73

Sancisi R., Fraternali F., Oosterloo T., van der Hulst T., 2008, A\&A Rev., 15, 189

Sault R. J., Teuben P. J., Wright M. C. H., 1995, in Shaw R. A., Payne H. E., Hayes J. J. E., eds, Astronomical Data Analysis Software and Systems IV Vol. 77 of Astronomical Society of the Pacific Conference Series, A Retrospective View of MIRIAD. pp 433-+

Schaap W. E., Sancisi R., Swaters R. A., 2000, A\&A, 356, L49

Shapiro P. R., Field G. B., 1976, ApJ, 205, 762

Sofue Y., Rubin V., 2001, ARA\&A, 39, 137

Swaters R. A., Balcells M., 2002, A\&A, 390, 863

Swaters R. A., Sancisi R., van der Hulst J. M., 1997, ApJ, 491, 140

Valdes F., , 1995, Guide to the HYDRA Reduction Task DOHYDRA

van der Hulst J. M., Terlouw J. P., Begeman K. G., Zwitser W., Roelfsema P. R., 1992, in Worrall D. M., Biemesderfer C., Barnes J., eds, Astronomical Data Analysis Software and Systems I Vol. 25 of Astronomical Society of the Pacific Conference Series, The Groningen Image Processing SYstem, GIPSY. pp 131-+

van der Hulst J. M., van Albada T. S., Sancisi R., 2001, in J. E. Hibbard, M. Rupen, \& J. H. van Gorkom ed., Gas and Galaxy Evolution Vol. 240 of Astronomical Society of the Pacific Conference Series, The Westerbork HI Survey of Irregular and Spiral Galaxies, WHISP. pp 451-+ van der Kruit P. C., 2007, A\&A, 466, 883

van Zee L., 2000, AJ, 119, 2757

van Zee L., 2001, AJ, 121, 2003

Westmeier T., Braun R., Thilker D., 2005, A\&A, 436, 101

This paper has been typeset from a $\mathrm{T}_{\mathrm{E}} \mathrm{X} / \mathrm{LT}_{\mathrm{E}} \mathrm{X}$ file prepared by the author. 